\author{
Markus Raab \\ German Sport University, Germany, Institute of Psychology \\ London South Bank University, United Kingdom, School of Applied Sciences
}

Motor Heuristics and Embodied Choices: How to Choose and Act

submitted to Current Opinion in Psychology 2000 words

Address: Deutsche Sporthochschule Köln

Institute of Psychology

Am Sportpark Müngersdorf 6

50933 Köln, Germany

raab@dshs-koeln.de

Tel. $++49 / 221 / 49825491$

Fax $++49 / 221 / 49828320$ 
Abstract

Human performance requires choosing what to do and how to do it. The goal of this theoretical contribution is to advance understanding of how the motor and cognitive components of choices are intertwined. From a holistic perspective I extend simple heuristics that have been tested in cognitive tasks to motor tasks, coining the term motor heuristics. Similarly I extend the concept of embodied cognition, that has been tested in simple sensorimotor processes changing decisions, to complex sport behavior coining the term embodied choices. Thus both motor heuristics and embodied choices explain complex behavior such as studied in sport and exercise psychology. 


\section{Motor Heuristics and Embodied Choices: How to Choose and Act}

Michael Jordan, a famous basketball player, attempted 24,537 field goals in his career and hit roughly $50 \%$ of them $(.497)$. When he moved to the free-throw line, about $80 \%$ of 7,327 attempts were successful (.835). Field goals require a choice of shooting or passing that needs to be made in milliseconds from any number of positions in the field, under defensive pressure, and with uncertainty associated with the choice. In free throws, there is no choice between options; the situation is much more constant and less likely to fail given the base rates.

In established research programs, cognitive science and movement science have independently tried to explain the making of choices, such as selecting between two or more options. Whereas cognitive science has focused on what to do, movement science has focused on how to do it. This separation is falling out of favor [1], and the term "embodiment" has been introduced to bridge the two disciplines. Embodiment describes the link between sensorimotor and cognitive processes. It has appeared in more than 10,000 papers, with more than three new papers per day published in 2015 (Web of Science; URL: https://login.webofknowledge.com). Terms such as "embodied cognition" illustrate that the research gap is beginning to close (for an overview see [2]).

The rationale for describing choices and movements in one framework is based on a holistic approach to describing behavior [3]. Although it can be useful to divide the scientific exploratory work between disciplines such as movement science and cognitive science, it can limit the ability to describe complex training or competition behavior in disciplines such as human movement science [4]. Likewise in science, experimentally testing perceptual factors that influence performance is often detached from controlling cognitive or emotional factors and, for internal validity, 
often reduced to simple stimuli and simple key-press responses. This commonly accepted "divide and conquer" approach in science may be unsuitable for exploring complex behavior such as Michael Jordan's decision to shoot or pass [5].

To provide a starting point for a future research program combining cognitive and movement science, I have coined two terms, described in the following sections. "Motor heuristics" borrows from the cognitive science research describing how people choose under limited time and distribute their cognitive resources. It stresses that the motor system does explain part of the choices. The concept of "embodied choices" is derived from movement science research programs that describe how people control their movements in various situations, influencing their choices.

\section{Motor Heuristics}

I define a motor heuristic as a simple rule of thumb that allows an athlete to choose between options (here movements) to satisfy the current needs in a situation. This concept is based on the notion of simple heuristics [6], which have been used mainly in cognitive tasks such as the city-size question (e.g., which of two German cities, Berlin or Hamburg, has more inhabitants?). Heuristics are composed of search, stop, and decision rules: For instance, in the city comparison, search might start with the most valid cue, such as whether one of the cities is a capital. If one of the options is positive on that cue and the other not, the decision maker stops considering further cues and decides for the city with the positive value on that cue (here, Berlin). For motor heuristics, a holistic approach would add an execution rule. In Michael Jordan's case, he needs to search for information to decide between passing and shooting and to stop this information search when a cue is positive for shooting, such as his distance from the basket or the closest defensive player. Michael further needs to 
decide how he should produce the shooting movement given the distance or the behavior of the defensive player.

Motor heuristics are therefore able to capture the search for information and the choice of what to do and how to do it [7]. Recently this concept of simple heuristics in cognitive tasks has been transferred to the scientific study of sports behavior. This allows for describing and explaining complex behavior not as a list of separate abilities but rather in terms of heuristics that integrate cognition and action components of complex behavior (for reviews see $[8,9]$ ). For another example consider table tennis [7]: A player has only milliseconds to process the visual information about where the opponent has placed the ball and with what kind of spin. The player decides whether to prepare a forehand or backhand stroke and how to realize that movement to counteract the spin with temporal and spatial precision. Such a complex movement can be separated into deciding what to do (forehand vs. backhand) and how to do it (topspin, underspin). Heuristics can define a search rule (movement rotation of the bat), a stop rule (no further information needed), a decision rule (if right-handed, use forehand if ball falls to the right of your midline), and an execution rule (rotate opposite to the direction of spin to counteract the spin).

\section{Embodied Choices}

I define embodied choices in the same vein as motor heuristics, as rules of thumb that are useful when limited time and resources force athletes to decide quickly between two or more options. However in the context of real behavior, I argue that one cue that has so far been neglected for such choices is the human body and its stored sensorimotor experiences (but see [10-12]). Consider again Michael Jordan's options: Embodied choice models assume that the sensorimotor system itself provides cues for choices. For instance, the perceptual ability to resolve details in dynamic 
situations of moving players and objects is called dynamic visual acuity (DVA). In basketball, shooting performance has been shown to correlate with DVA in some studies (e.g., [13]). If that DVA changes due to fatigue or other factors, it may serve as a cue to change the decision of whether to pass or shoot. Other cues often used to explain athletes' choices refer to cost-value analysis or cognitive examination of complex calculations of expected values, but these seem to be less important (see detailed comparisons of different models in [10]).

For embodied choices, the execution as described in motor heuristics is thus not only the end product of a cognitive choice between two options but itself serves as a cue about whether one or the other option can be processed. An important implication is that in two-phase models it is assumed that choosing what to do and how happens sequentially. However it seems likely that these processes run in parallel, as supported by neurophysiological and animal research (e.g., [14]). Such arguments have been tested in much simpler behavior [10] and recently the concept has been extended and revised for skill acquisition in sport performance [15].

\section{Integrating Motor Heuristics and Embodied Choices}

A benefit of introducing two concepts-motor heuristics and embodied choices - to describe human performance is that motor heuristics focus much more on the execution than embodied choices and embodied choices focus much more on how are options generated [7]. Theoretically motor control can be understood in terms of decision making (e.g., [16]), but as illustrated in the Michael Jordan example, an explanation of the shoot-or-pass decision or a free throw movement requires different descriptions of cognitive and action components (see Figure 1).

insert Figure 1 about here 
Integrating the two concepts allows for describing and explaining a range of behaviors in sports psychology, from very routine motor behavior that is performed in milliseconds to complex behaviors that are enacted over longer periods of time. Empirical evidence for heuristics has been focused so far on athletes' behavior (for a recent summary see [9]). Empirical evidence for specific heuristics and tests in independent labs have supported some of the descriptive and exploratory power (e.g., [17]). Further, those heuristics have also been specified in computational models (e.g., [18]), longitudinal development, and emotional choices and related to biomarkers (see [8] for an overview). Finally specific heuristics that have been developed in sports science have been transferred to other disciplines and tasks such as navigation, law, medicine, and consumer choice (see [19] for an example). There are a few limitations to the approach: Systematic applications describing how motor heuristics are learned are still needed, comparisons to alternative models are scarce (but see [18] in sports or [10] for hand movements), and the concept does not transfer well to person-specific questions, such as testing the developmental nature of motor heuristics or embodied choices over the lifespan (e.g., [20]) or applying it to other choices in sport, for example, by coaches, referees, fans, or managers (e.g., [21]).

What are the benefits of applying the holistic approach of motor heuristics and embodied choices to an applied field such as sports psychology? I believe there are advantages on three levels: theory, method, and practice. Theoretically, a holistic approach would not describe, explain, and predict sports behavior in the classic language of building blocks or performance (e.g., perception, cognition, emotion, action) but rather would integrate the behaviors into heuristics that describe internal or external search (memory, perception), stopping information search (cognitive processes, situated actions), and making a choice (option generation, action selection) 
in concepts of motor heuristics and embodied choices. Whether theoretically motor heuristics are applied to understand the spatial and temporal precision of the movement or embodied choices are applied to understand the situational and personal processes of a long-term choice is simply task- and person-specific. The holistic approach thus allows capturing the interaction of the multiple and parallel processes that are present in complex behavior, with all the consequences that may follow for designs and tests. However, describing complex behavior and multiple interactions itself does not imply complex models, as the power of simplicity is a hallmark of heuristics in general and motor heuristics specifically (e.g., [9]).

Methodologically, a list of processes would not be sufficient to satisfy a holistic approach. Rather, one advantage of applying motor heuristics and embodied choices is being able to align tests and dependent variables that too often have been treated independently. For instance, gaze data that provide information about external search should be combined with an option-generation paradigm to measure internal cognitive processes, with the action selection and execution itself. The technology to synchronize and use mobile eye trackers and mobile electroencephalography while simultaneously measuring kinematics exists but is not yet systematically used in most laboratories. Recent attempts that go beyond correlating dependent variables to measure the dynamics of internal and external search and complex behavior indicate that research is moving in this direction (e.g., [22,23]).

On the practical side, if validated in the future, a holistic approach would suggest alternatives for training and competition. For instance, practicing individual abilities would be replaced by training specific heuristics, and diagnostics would be based not on profiles of cognitive or motor components but on whether and how well athletes apply motor heuristics or embodied choices (e.g., [24]). Whether feedback, 
instructions, and training methods will change is too far in the future to be foreseen and requires empirical testing.

In conclusion, merging cognitive science and movement science into one holistic approach to describe complex actions has not yet been attempted. The judgment and decision-making research on simple heuristics is promising, if execution rules that describe movement-related interactions with the environment are added. The movement science research on embodied cognition is promising, if its adds combined descriptions of decision and execution rules. I argued here that motor heuristics and embodied choices are concepts that need to be further developed, tested, and applied in sport and exercise psychology. 


\section{Acknowledgments}

I would like to thank Rouwen Canal-Bruland for the term "motor heuristics," which came up in a project meeting discussion; the term "embodied choices" was used in Raab, Johnson, and Heekeren [25]. 


\section{References}

**[1] Rosenbaum DA: The Cinderella of psychology: the neglect of motor control in the science of mental life and behavior. Am Psychol 2005, 60:308-317.

This paper indicates the neglect of motor control in psychological research and indicates why that is important.

**[2] Fischer MH, Coello Y (Eds): Foundations of Embodied Cognition: Volume 1. Perceptual and Emotional Embodiment. Taylor \& Francis; 2016.

The book is a comprehensive summary of embodied cognition literature.

[3] Bergman R, Cairns R, Nielson LG, Nystedt L (Eds): Developmental Science and the Holistic Approach. Erlbaum; 2000.

[4] Glazier PS: Towards a grand unified theory of sports performance. Hum Mov Sci 2015, http://dx.doi.org/10.1016/j.humov.2015.08.001.

*[5] Raab M, Lobinger B, Hoffmann S, Pizzera A, Laborde S (Eds): Performance Psychology. Perception, Action, Cognition, and Emotion. Academic Press; 2016.

This book integrates from a performance psychology perspective concepts such as perception, action, cognition and emotion in theory, methodology and applied research in sport, music and movement science.

**[6] Gigerenzer G, Todd PM, the ABC Research Group: Simple Heuristics That Make Us Smart. Oxford University Press; 1999.

This book describes bounded rationality and ecological rationality concepts using fast and frugal heuristics in many different domains.

[7] Raab M, Masters R, Maxwell J: Improving the how and what decisions of table tennis elite players. Hum Mov Sci 2005, 24:326-344. 
[8] Raab M: Simple heuristics in sports. Int Rev Sport Exerc Psychol 2012, 5:104120.

*[9] Raab M, Gigerenzer G: The power of simplicity: a fast-and-frugal heuristics approach to performance science. Front Psychol 2015, doi:10.3389/fpsyg.2015.01672/full.

This paper summarizes the simple heuristic approach for performance science focusing on applications in medicine, sport, music and arts.

[10] Lepora NF, Pezzulo G: Embodied choice: how action influences perceptual decision making. PLoS Comput Biol 2015, doi:10.1371/journal.pcbi.1004110

[11] Reimann M, Feye W, Malter AJ, Ackerman JM, Castaño R, Garg N, Kreuzbauer R, Labroo AA, Lee AY, Morrin M et al.: Embodiment in Judgment and Choice. J Neurosci Psychol Econ, 2012, 5:104-123. doi:10.1037/a0026855.

[12] Wyer RS Jr: Priming Decisions and Motor Behavior. Cogn Psychol 2016, http://dx.doi.org/10.1016/j.copsyc.2016.06.002.

[13] Beals RP, Mayyasi A, Templeton AE, Johnston WL: The relationship between basketball shooting performance and certain visual attributes. $A m J$ Optom Arch Am Acad Optom 1971, 48: 585-590.

**[14] Cisek P, Pastor-Bernier A: On the challenges and mechanisms of embodied decisions. Philos Trans R Soc Lond B Biol Sci 2014, 369: 20130479. http://dx.doi.org/10.1098/rstb.2013.0479.

This paper describes the basic mechanisms of sensorimotor decisions that are embodied.

[15] Raab M: SMART-ER: a situation model of anticipated response consequences in tactical decisions in skill acquisition-extended and revised. Front Psychol 2015, 5: doi:10.3389/fpsyg.2014.01533 
**[16] Wolpert DM, Landy MS: Motor control is decision-making. Curr Opin Neurobiol 2012, 22:996-1003. doi:10.1016/j.conb.2012.05.003.

This paper integrates decision making with motor control in a new model.

[17] Hepler TJ, Feltz DL: Take the first heuristic, self-efficacy, and decisionmaking in sport. $J$ Exp Psychol Appl 2012, 18:154-161.

[18] Glöckner A, Heinen T, Johnson J, Raab M: Network approaches for expertdecision in sports. Hum Mov Sci 2012, 31:318-333.

[19] Conlin JA: Getting around: Making fast and frugal navigation decisions. In Mind and Motion: The Bidirectional Link Between Thought and Action (Progress in Brain Research, Vol. 174). Edited by Raab M, Johnson J, Heekeren H. Elsevier; 2009:109-117.

[20] Löffler J, Cañal-Bruland R, Raab M: A lifespan perspective on embodied cognition. Front Psychol 2016, 7:845.

[21] Csapo P, Avugos S, Raab M, Bar-Eli M: How should "hot" players in basketball be defended? The use of fast-and-frugal heuristics by basketball coaches and players in response to streakiness. $J$ Sports $S c i$ 2015, 33:1580-1588. doi:10.1080/02640414.2014.999251.

[22] Canal-Bruland R, Mann DL Time to broaden the scope of research on anticipatory behaviour: a case for the role of probabilistic information. Front Psychol 2015, 6:1518. doi:10.3389/fpsyg.2015.01518.

[23] Raab M, Johnson J: Option-generation and resulting choices. J Exp Psychol Appl 2007, 13:158-170.

[24] de Oliveira RF, Lobinger BH, Raab M: An adaptive toolbox approach to the route to expertise in sport. Front Psychol 2014, 5:doi:10.3389/fpsyg.2014.00709 
**[25] Raab M, Johnson J, Heekeren H (Eds): Mind and Motion: The Bidirectional Link Between Thought and Action (Progress in Brain Research, Vol. 174). Elsevier; 2009.

This book describes how actions influence cognitive processes and vice versa. 
Figure 1. Integrating motor heuristics and Embodied Choices for how to choose and act in the domain of sport psychology 


\section{Motor Heuristics and Embodied Choices} How to choose and act

Motor Heuristics

Search-stop-decision-

execution rules

What movement to execute

and how to execute it

Embodied Choices

Search-stop-decision rules

include cues from the body

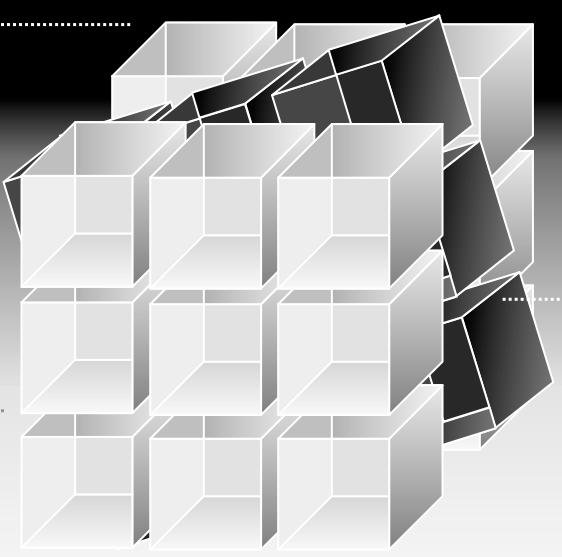

How to choose and act in

sportpsychology 